\title{
Mécanismes de déformation, d'endommagement et de rupture de joints collés
}

\author{
Costantino Creton ${ }^{\mathrm{a}}$ \\ Laboratoire PPMD, ESPCI, 10 rue Vauquelin, 75231 Paris Cedex 05, France
}

Reçu le 2 juillet 2004, accepté le 5 novembre 2004

\begin{abstract}
Résumé - Dans le collage, deux pièces sont assemblées par le dépôt d'une couche fine (typiquement 20-100 $\mu \mathrm{m}$ ) d'adhésif qui peut être de fort module (GPa) ou de faible module (MPa) suivant le type d'assemblage souhaité. Afin d'évaluer la tenue mécanique de cet assemblage, il doit être soumis à un test destructif destiné à rompre le joint adhésif et à séparer les deux pièces. Comme le matériau composant l'adhésif est invariablement un polymère, il est important d'étudier la résistance à la rupture d'une couche de polymère confinée. Dans cet article nous discutons brièvement l'état de l'art sur la rupture des polymères vitreux et des élastomères dans une géométrie confinée. Dans ce type de géométrie plusieurs effets spécifiques sont observés lors de la rupture du joint : d'une part les contraintes internes dans la couche jouent un rôle essentiel et peuvent profondément modifier les mécanismes de rupture, d'autre part le fort degré de triaxialité de la contrainte favorise la nucléation de cavités. Cette cavitation, favorisée pour tous les types de polymères, est particulièrement prononcée pour les élastomères.
\end{abstract}

Mots clés : Fracture / cavitation / adhésion / interface / polymère

\begin{abstract}
Deformation, yield and fracture mechanisms of adhesive joints. When two surfaces are bonded together by an adhesive, a thin layer of adhesive (typically 20-100 $\mu \mathrm{m}$ ), with a high $(\mathrm{GPa})$ or low ( $\mathrm{MPa}$ ) elastic modulus is deposited between the two surfaces to assemble. In order to evaluate the mechanical strength of the bond, the only reliable way is to perform a destructive test aimed at the fracture of the joint and eventual separation of the two surfaces. Since the adhesive components are invariably polymers, it is essential to study the behaviour of polymers in a confined geometry. In this case several effects, very specific to thin confined films, are present. In particular the residual stresses can be more prominent and modify the type of plasticity mechanisms which are observed upon fracture of the joint. For soft adhesives (elastomeric) the confinement strongly increases the degree of triaxiality of the applied stress, which favors the nucleation and the growth of cavities. This cavitation is favored by the confinement for all types of polymers but it is the elastomers which are the most sensitive to it.
\end{abstract}

Key words: Fracture / cavitation / adhesion / interface / polymer

\section{Introduction}

La plupart des colles et matériaux collants sont des polymères. En effet la fonction d'un adhésif requiert d'être un liquide pour former le contact, même sur une surface rugueuse, et d'être un solide pour résister à l'application d'une contrainte sur l'interface une fois le joint formé. Cette transition peut se faire par un changement de température, par une réaction chimique, par l'exposition aux UV ou encore par un choix judicieux des propriétés viscoélastiques du polymère. Seuls les polymères peuvent passer relativement facilement à température ambiante d'un état solide à un état liquide.

\footnotetext{
a Auteur correspondant : Costantino.Creton@espci.fr
}

D'autre part l'adhésif est pratiquement exclusivement utilisé en couches minces sollicitées en cisaillement, ce qui impose un certain nombre de contraintes supplémentaires. Typiquement un joint adhésif a une épaisseur de 10 à $100 \mu \mathrm{m}$ et le matériau adhésif est plus ductile que les matériaux de part et d'autre du joint. La conception du joint est importante pour éviter les concentrations de contrainte pouvant solliciter l'adhésif en traction pure.

Les adhésifs se divisent en deux classes principales que nous allons discuter :

- Les adhésifs structuraux tels que les époxy se distinguent par un module d'élasticité élevé caractéristique des polymères vitreux de l'ordre de 3-4 GPa, par une transition liquide-solide qui se fait 
généralement par réaction chimique et par un seuil de plasticité de l'ordre de 20-50 MPa.

- Les adhésifs souples ont des modules d'élastomères (0,1-10 MPa), une transition liquide-solide qui peut se faire par réaction chimique mais aussi par cristallisation ou changement de phase. Ils sont déformables et peuvent donc accommoder des déformations importantes de façon réversible.

Bien que dans le langage courant on parle bien sûr d'adhésifs, ce qui met l'accent sur la formation du contact (l'adhésion), en réalité l'adhésion ne peut pas être évaluée sans une destruction de ce dit contact. Si les liaisons ou interactions chimiques à l'interface sont fortes, aucune séparation interfaciale (rupture de ces liaisons) n'est possible sans une déformation plastique importante d'un volume de polymère immédiatement adjacent à l'interface ce qui induit un endommagement important de la couche d'adhésif.

Le caractère confiné de la couche adhésive modifie significativement les champs de contrainte appliqués à l'interface et implique généralement un haut degré de triaxialité. Cette triaxialité, à son tour, influence très significativement les mécanismes de plasticité des polymères que nous allons discuter.

\section{Cas de la rupture des polymères thermoplastiques et thermodurcissables (solides)}

Les adhésifs structuraux peuvent être appréhendés par la compréhension des mécanismes de rupture et d'adhésion des polymères vitreux. La rupture des polymères vitreux se fait généralement de façon fragile ou faiblement ductile et peut être traitée par la mécanique linéaire de la rupture qui suppose un comportement globalement élastique du matériau. On distingue trois échelles spatiales importantes dans un problème d'adhésion de polymères vitreux :

- L'échelle macroscopique de l'échantillon qui détermine le type de chargement et en particulier le degré de mixité de ce chargement (mode I ou mode II ou mode mixte). Cette échelle est millimétrique ou centimétrique pour la plupart des cas.

- L'échelle de la zone de déformation plastique en pointe de fissure. Les polymères ne se cassent jamais de façon totalement fragile tel le verre inorganique. Une fracture est toujours précédée d'une zone plastifiée dont l'épaisseur caractéristique peut varier entre quelques centaines de nanomètres et quelques dizaines de micromètres. La forme et la taille de cette zone plastique déterminent l'énergie dissipée lors de la fracture et dépendent de façon importante des propriétés du polymère dans le domaine de l'écoulement plastique.

- L'échelle moléculaire du plan de rupture. En effet pour propager une fissure dans un matériau, des liaisons chimiques doivent être cassées. Il est important de connaître l'organisation des chaînes de polymère dans le plan de rupture pour comprendre les mécanismes de transferts de contrainte et de rupture.

\section{Interfaces entre polymères vitreux}

Les interfaces entre polymères vitreux ont permis d'élucider beaucoup de points concernant le couplage entre la structure chimique de l'interface et les propriétés de fracture. En effet grâce à l'utilisation de systèmes modèles, il a été possible de découpler la structure et l'organisation macromoléculaire à l'interface des propriétés de déformation plastique des polymères de part et d'autre de l'interface, ce qui n'est pas possible dans le cas d'un polymère homogène. Dans la suite de cette partie nous allons discuter les interfaces entre polymères vitreux.

La plupart des polymères vitreux se fracturent de façon relativement fragile et la fissure se propageant est précédée d'une zone plastique allongée et peu dense formée de fibrilles. Ces zones fibrillaires sont appelées craquelures et ont été beaucoup étudiées dans les années 80 [1,2]. Pour que ces zones fibrillaires puissent être observées, trois étapes sont importantes, la nucléation ou la croissance de cavités dans le polymère vitreux, l'évolution de ces cavités vers une structure de fibrilles orientées et puis l'épaississement de ces craquelures par l'incorporation progressive de matière non plastifiée à partir d'une zone intermédiaire d'une épaisseur de quelques nanomètres où les chaînes polymères s'orientent [1]. Une image de microscopie électronique à transmission d'une craquelure partiellement fracturée [3] en tête de fissure est montrée sur la figure 1.

Sur cette image, on peut voir l'interface fracturée et la craquelure formée uniquement d'un côté de l'interface, par plastification et orientation du polymère possédant le plus faible seuil de plasticité. Le modèle de zone cohésive de Dugdale [4] a été utilisé par Brown [5] pour prédire la relation suivante entre l'épaisseur maximale $h$ de la craquelure, la contrainte de formation de la craquelure $\sigma_{\mathrm{c}}$ et l'énergie de fracture $G_{\text {Ic }}$ :

$$
G_{\text {Ic }}=h \sigma_{\mathrm{c}}\left(1-\frac{1}{\lambda}\right)
$$

Dans cette expression, $\lambda$ est le facteur d'élongation des chaînes polymères dans la zone orientée et varie typiquement entre 3 et 8 . Ce modèle permet de bien décrire une zone de déformation fibrillaire soumise à une contrainte normale constante, au sein d'un milieu élastique homogène. Par contre, les fibrilles sont modélisées comme des ponts élastiques parallèles. Une série d'expériences comparant directement les valeurs de $G_{\text {Ic }}$ obtenues par une méthode de mécanique de la rupture et celles obtenues par visualisation par microscopie électronique à transmission de l'épaisseur de la zone plastique et en utilisant l'équation (1) a montré une très bonne corrélation linéaire entre ces deux valeurs confirmant des résultats antérieurs obtenus par interférence optique [3]. Il faut noter toutefois que l'équation (1) ne contient pas d'informations sur la structure moléculaire à l'interface. 


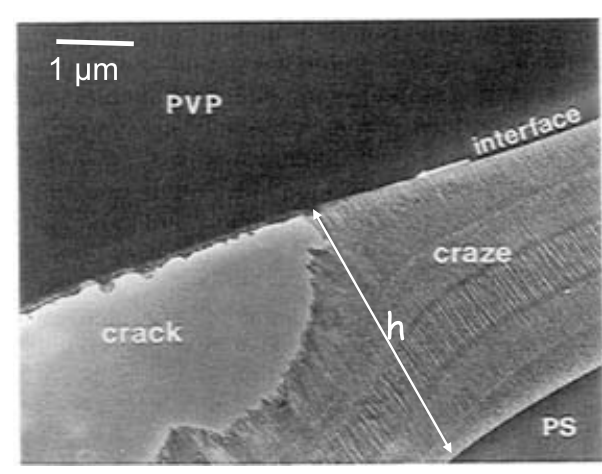

Fig. 1. Image de microscopie électronique à transmission d'une zone de craquelures à l'interface entre deux polymères vitreux. L'image montre clairement l'interface, la partie fracturée (crack) et la zone endommagée de polymère orienté (craze). Image tirée de la référence [3].

L'intégration de la structure moléculaire dans le modèle a été faite en 1991 par Brown [5] qui a postulé que la zone de polymère hautement orientée formant les fibrilles, visible sur la figure 1, pouvait être en fait modélisée par un matériau élastique anisotrope et que par conséquent un transfert de contrainte pouvait s'effectuer vers les dernières fibrilles non fracturées. Le résultat de ce modèle est une prédiction, qui, cette fois, relie directement la densité de liaisons covalentes à l'interface avec la valeur de $G_{\mathrm{c}}$ macroscopique qui peut être obtenue par un test de mécanique de la rupture classique comme le clivage.

Deux résultats importants et vérifiables expérimentalement ressortent de ce modèle. Pour des interfaces résistantes, l'épaisseur maximale $h_{\mathrm{f}}$ de la couche déformée plastiquement en tête de fissure est donnée par [5] :

$$
h_{\mathrm{f}} \propto\left(\frac{\sigma_{\mathrm{int}}}{\sigma_{\mathrm{c}}}\right)^{2}
$$

et le taux de restitution d'énergie critique est donné par :

$$
G_{\mathrm{c}} \propto \frac{\left(\sigma_{\mathrm{int}}\right)^{2}}{\sigma_{\mathrm{c}}}
$$

où $\sigma_{\text {int }}$ est la contrainte maximale que peut supporter l'interface (à l'échelle locale de la zone plastique) avant rupture des liaisons chimiques et $\sigma_{\mathrm{c}}$ est la contrainte critique macroscopique de formation de la zone plastique orientée. Il est important de noter que, pour que la zone plastique existe, $\sigma_{\text {int }}$ doit être au moins 1,2 fois supérieur à $\sigma_{\mathrm{c}}$ et que, pour une adhésion forte, il peut atteindre 3 fois $\sigma_{\mathrm{c}}$.

Ces résultats ont été vérifiés lors d'expériences de fracture d'interfaces entre polymères vitreux pour lesquelles $\sigma_{\text {int }}$ est donné par $f_{\mathrm{b}} \Sigma$, c'est-à-dire par le produit de la force nécessaire à casser une liaison $\mathrm{C}-\mathrm{C}$ par la densité surfacique des liaisons $\mathrm{C}-\mathrm{C}$ traversant l'interface, paramètre aisément contrôlable lorsque l'adhésion vient de connecteurs moléculaires.

À la suite de cette première modélisation, d'autres variantes et raffinements du modèle ont été développés par des mécaniciens de la rupture $[6,7]$.
À la suite de ces premières expériences sur des interfaces «modèle » renforcées par des connecteurs à l'interface, le modèle a été validé aussi pour l'analyse d'interfaces entre polymères partiellement miscibles, ce qui a permis de mettre en évidence qu'une interpénétration des chaînes polymères de l'ordre de 5 à $10 \mathrm{~nm}$ suffit pour créer des enchevêtrements et atteindre une plastification de la tête de fissure et donc une énergie de rupture élevée de quelques centaines de $\mathrm{J} / \mathrm{m}^{2}[8]$.

D'autre part une série d'expériences sur des interfaces entre le polyamide 6 (PA6) et le polypropylène (PP) renforcé par des copolymères de PA-6-PP formés in situ ont montré que le rôle du seuil de plasticité $\sigma_{\mathrm{c}}$ était également correctement prédit par ce modèle. En effet lorsque le polypropylène isotactique est remplacé par un alliage biphasique de PP et de caoutchouc EPDM, de seuil de plasticité quatre fois inférieur à celui du PP, les valeurs de $G_{\mathrm{C}}$ mesurées à $\sigma_{\text {int }}$ équivalent sont quatre fois plus élevées et les valeurs de $h_{\mathrm{f}}$ sont seize fois plus élevées pour le $\mathrm{PP} / \mathrm{EPDM}[9,10]$ en parfait accord avec l'équation (3). Des observations de la zone plastique par microscopie électronique à transmission ont permis de confirmer que la zone plastique était bien une zone formée de fibrilles de polymère orienté.

\section{Effets de confinement sur la fracture des joints adhésifs}

Une question intéressante du point de vue pratique est l'effet de la triaxialité sur les mécanismes de rupture des polymères. En effet, contrairement aux métaux, beaucoup de polymères sont capables, à température ambiante, de former des cavités lorsqu'ils sont soumis à une contrainte hydrostatique négative. Ce mécanisme de cavitation, qui n'intervient qu'en traction, se produit pour un seuil qui est généralement nettement inférieur au seuil de plasticité classique $\sigma_{y}$ observé par exemple lors de tests de compression uniaxiale.

Il est bien évident que la rupture d'un polymère dans des géométries telles que celles du joint adhésif (film mince entre deux surfaces rigides) favorise l'existence de fortes contraintes triaxiales. Une forte sensibilité à ces contraintes triaxiales serait donc une caractéristique peu souhaitable pour un adhésif. Typiquement les adhésifs structuraux sont des polymères, où les chaînes polymères sont fortement enchevêtrées ou réticulées chimiquement, ce qui inhibe la cavitation [2].

Par contre, il est intéressant de noter que dans ces conditions de forte triaxialité, certains polymères et notamment les polymères semi-cristallins, se fracturent par formation de craquelures amorcées par de la cavitation, alors qu'ils se déforment par formation de bandes de cisaillement lors d'une traction uniaxiale $[11,12]$.

Un autre aspect qu'il est important de noter est l'importance des contraintes internes. En effet une couche confinée est particulièrement affectée par une différence de coefficient d'expansion thermique avec les matériaux formant les deux surfaces de part et d'autre de la couche. 


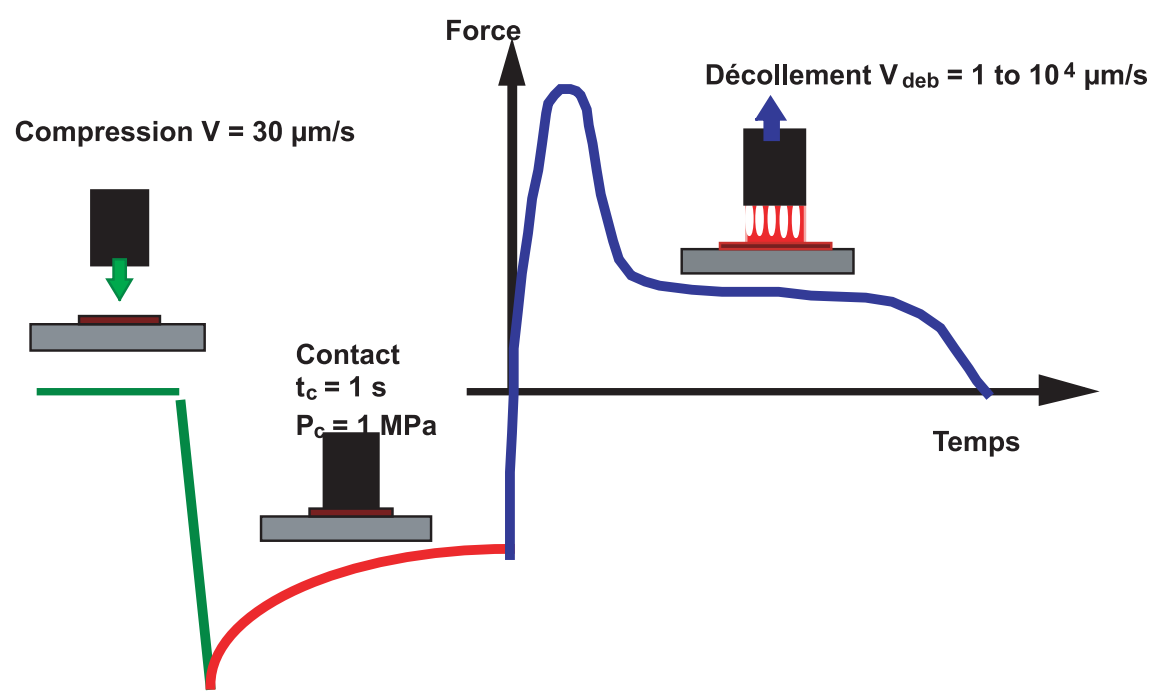

Fig. 2. Schéma d'une expérience de «tack» effectuée avec un poinçon plan sur une couche d'adhésif.

Si la formation de la couche confinée (et c'est souvent le cas pour les adhésifs), implique des variations importantes de température, le résultat final est une couche précontrainte. Ces contraintes de cisaillement se superposeront aux contraintes imposées lors du test de fracture et peuvent avoir deux effets importants : d'une part ils peuvent considérablement fausser la mesure intrinsèque de l'énergie élastique restituée au système [13] et d'autre part ils peuvent modifier la mixité de mode en tête de fissure et modifier ainsi les mécanismes de plasticité observés lors de la fracture. En particulier pour le cas des polymères vitreux, des phénomènes de bifurcation de fissures hors du plan de l'interface (dans la couche confinée) liés à des modifications fines de mixité de mode ont été observés [14].

\section{5 Élastomères sous forte triaxialité et cavitation}

Une autre catégorie de polymères très sensibles au degré de triaxialité de la contrainte appliquée est celle des élastomères et gels de polymères. Ces matériaux ont la particularité d'avoir un module de cisaillement (généralement compris entre 0,01 et $5 \mathrm{MPa}$ ) beaucoup plus faible que leur module de compressibilité (de l'ordre du GPa pour tous les solides dont les liaisons chimiques sont de type Van der Waals). Ce module de cisaillement faible est d'origine entropique et est lié à la déformation des chaînes polymère hors de leur état d'équilibre.

De ce fait, ils sont particulièrement sensibles au degré de triaxialité de la contrainte et peuvent caviter facilement lorsqu'ils sont soumis à des contraintes de l'ordre de leur module de Young $[15,16]$.

Une géométrie bien adaptée à l'étude de l'effet du confinement sur les mécanismes d'endommagement et de rupture de ces polymères au-dessus de leur température de transition vitreuse est la géométrie du poinçon plan montrée schématiquement sur la figure 2. Dans cette géométrie, on forme le contact entre un film mince de polymère déposé sur un substrat rigide et la face plane d'un poinçon également rigide. L'enfoncement (ou le retrait) du poinçon applique un champ de contrainte à la couche confinée, dont la forme précise dépend du rapport $\alpha$ entre le rayon du contact $a$ et l'épaisseur de la couche $h$. Ce problème a été traité par plusieurs auteurs [17-20] et présente une particularité intéressante : pour un matériau incompressible, la forme du champ de contrainte présente trois extrema dont un maximum au centre pour un rapport $\alpha$ supérieur à 2 .

La géométrie du poinçon est représentée sur la figure 3 : pour une contrainte de traction moyenne $\sigma_{\text {avg }}$ appliquée sur le poinçon, la distribution de contrainte de traction sous le poinçon en fonction de $r$ est donnée par la figure 4 pour différentes valeurs de $\alpha$ [20]. Dans le cas limite d'une couche épaisse, on retrouve le résultat classique du massif semi-infini; pour $\alpha \rightarrow 0$, la contrainte sous le poinçon est donnée par [21] :

$$
\sigma_{z z}(r)=\frac{\sigma_{\mathrm{avg}}}{2\left[1-\left(r^{2} / a^{2}\right)\right]^{1 / 2}}
$$

ce qui signifie que la contrainte maximale est en bord de poinçon et qu'on s'attend à une propagation de fissure circulaire de l'extérieur du poinçon vers son centre. Par contre, pour des valeurs de $\alpha$ importantes, l'importance de la singularité diminue et devient négligeable. Le profil de contrainte (en dehors de la partie la plus proche du bord) est donné par [22,23] :

$$
\sigma_{z z}(r)=2 \sigma_{\text {avg }}\left[1-\left(r^{2} / a^{2}\right)\right]
$$

Un autre aspect important lié à l'action conjuguée du confinement et de la quasi-incompressibilité est la sensibilité très grande du profil de contrainte donné sur la figure 4 à la valeur précise du coefficient de Poisson. Pour un matériau complètement incompressible et une valeur de $\alpha$ grande, $\sigma_{z z}(r)$ est donné par l'équation (5) et présente une forme parabolique. Par contre, lorsque $\nu$ 


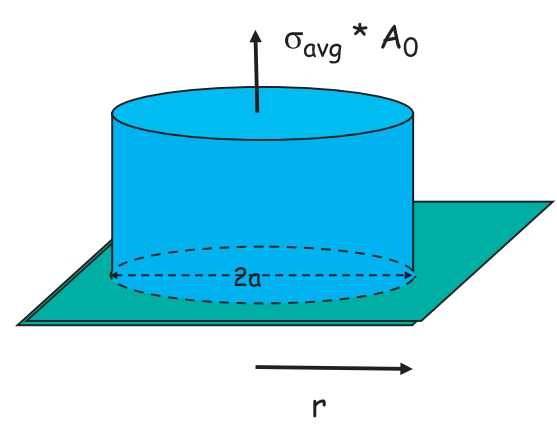

Fig. 3. Schéma du contact du poinçon avec le film adhésif montrant les grandeurs géométriques caractéristiques de l'expérience.

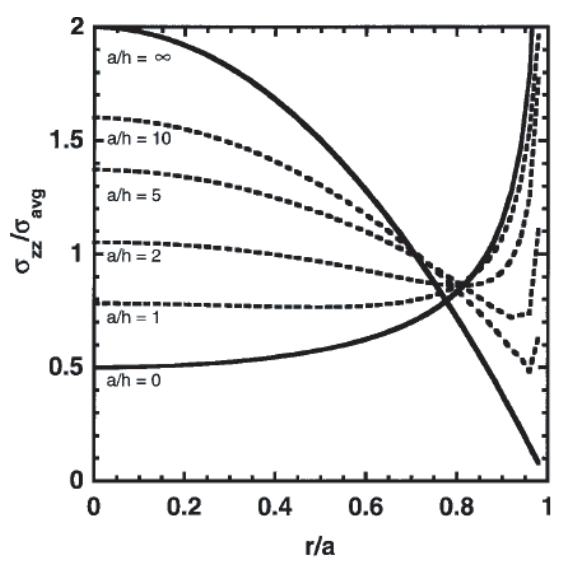

Fig. 4. Contrainte $\sigma_{z z}$ dans la direction normale au plan de l'adhésif, normalisée par la contrainte moyenne en fonction de la position radiale à partir du centre du poinçon pour le cas d'un adhésif incompressible et pour différentes valeurs de confinement $\alpha$. Figure tirée de la référence [20].

s'écarte même faiblement de 0,5 , le profil de contrainte s'aplatit de façon marquée [19] (Fig. 5). Cet aplatissement du profil de contrainte a des conséquences directes sur les mécanismes de fracture de la couche. En effet, le coefficient de Poisson est donné par :

$$
\nu=1 / 2-\frac{\mu}{2 K}
$$

avec $\mu$ le module élastique de cisaillement et $K$ le module de compressibilité. Par conséquent un écart à 0,5 de $\nu$ est directement relié au rapport $\mu / K$. Pour un rapport $\mu / K$ très faible, on s'attend à retrouver un profil de contrainte parabolique et donc une cavitation (sur un critère de contrainte maximale) qui commence au centre du poinçon. Par contre, pour un rapport $\mu / K$ plus élevé, on s'attend à un profil de contrainte plus aplati et à une cavitation distribuée de façon uniforme sur toute la surface du poinçon.

Ce résultat est observable expérimentalement (Fig. 6) pour deux polymères au-dessus de leur transition vitreuse soumis à une contrainte de traction : l'un, réticulé physiquement par des copolymères à bloc de type styrèneisoprène, cavite de façon homogène sur toute la surface de contact (A) alors que l'autre, un polyacrylate

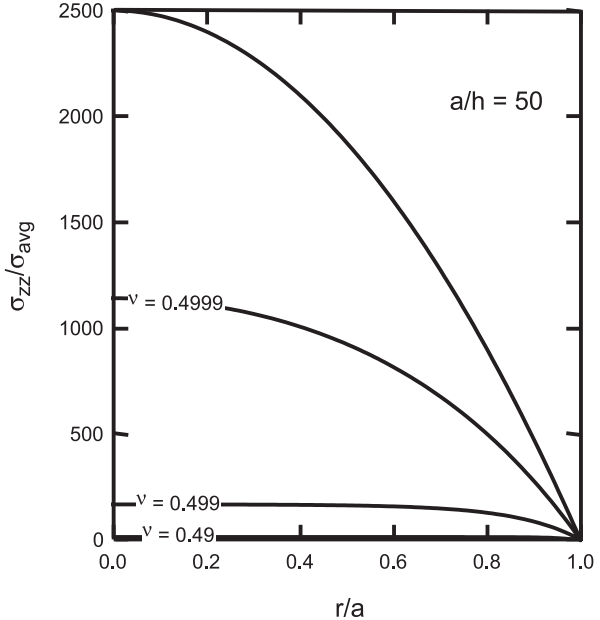

Fig. 5. Contrainte $\sigma_{z z}$ dans la direction normale au plan de l'adhésif, normalisée par la contrainte moyenne en fonction de la position radiale à partir du centre du poinçon pour le cas où $\alpha=50$ et pour différentes valeurs du coefficient de Poisson $\nu$. Figure tirée de la référence [19].

de n-butyle linéaire cavite préférentiellement au centre du contact (B). Cette différence peut être attribuée à une différence de module de cisaillement entre les deux polymères.

Refermons maintenant cette parenthèse et revenons aux implications du résultat central présenté sur la figure 4 , soit la modification du profil de contrainte pour une variation du paramètre $\alpha$ caractérisant le confinement. Si en compression cette modification du profil de contrainte n'a pas de conséquences visibles, ce n'est pas le cas en traction. En effet dans le cas de figure où le contact entre le poinçon plan et le film de polymère est adhésif, on peut observer expérimentalement des mécanismes de fracture du contact différents suivant le degré de confinement du film [24-26]. Pour un film non confiné $(a / h \rightarrow 0)$, la séparation se fait généralement par propagation d'une fissure amorcée en bordure du contact là où la concentration de contrainte est la plus élevée. Toutefois, lorsque l'épaisseur du film est réduite $(a / h$ grand), on observe que la séparation se fait par nucléation de cavités à l'intérieur de la surface de contact (Fig. 6a).

Cette transition d'un mécanisme de fracture à un mécanisme de cavitation a été décrite en détail dans quelques publications récentes $[19,26,27]$ et peut être expliquée qualitativement de la façon suivante : le critère de propagation de fracture interfaciale à partir du bord du contact peut être écrit, pour une couche élastique :

$$
G>G_{\mathrm{c}}
$$

avec $G$, le taux de restitution d'énergie et $G_{\mathrm{c}}$ le taux critique nécessaire à la propagation d'une fissure. Ce taux de restitution d'énergie par unité de surface correspond à l'énergie élastique restituée par le système lors de la création d'une surface fracturée d'aire $d A$. À même contrainte appliquée, plus la couche est mince, plus cette grandeur est petite. Comme la valeur critique $G_{\mathrm{c}}$ ne varie 

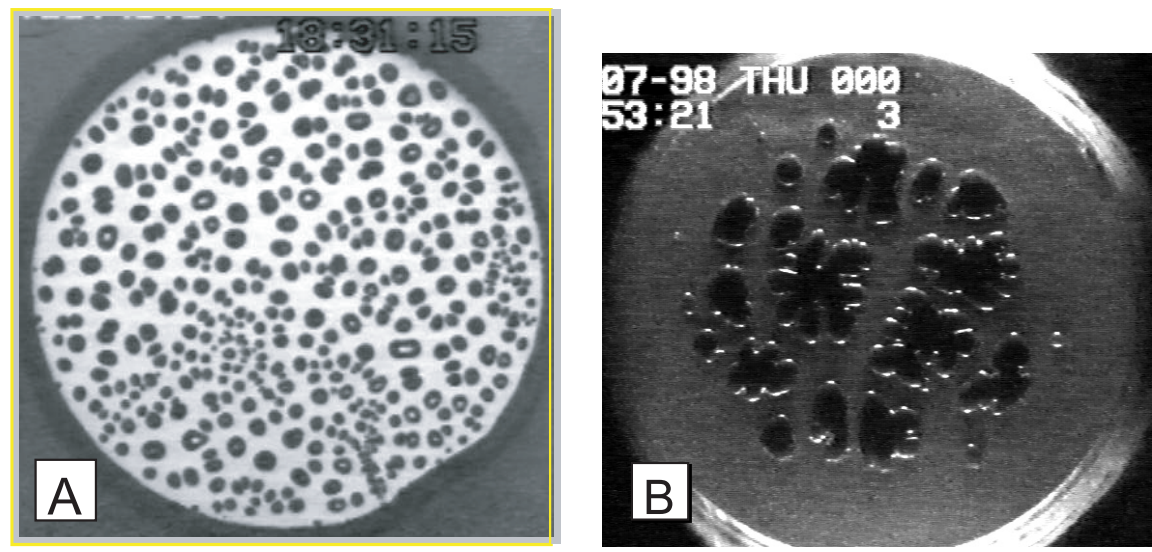

Fig. 6. Images du décollement d'un poinçon plan de deux adhésifs différents. Les images ont été prises au niveau du pic de la force de traction de la figure 2. Sur la figure A le diamètre du poinçon est de $6 \mathrm{~mm}$ et l'épaisseur de la couche est de $120 \mu \mathrm{m}$ alors que sur la figure B, l'épaisseur de la couche est de $100 \mu \mathrm{m}$ et le diamètre du poinçon est de $10 \mathrm{~mm}$. Les images sont tirées des références [29] et [30].

pas avec le confinement, puisqu'il s'agit d'une énergie dissipée par unité de surface créée, on arrive au résultat que la fissure partant du bord du contact est d'autant plus difficile à faire propager que la couche est confinée.

D'autre part, la croissance d'une cavité préexistante dans un milieu hyperélastique aura lieu si localement la contrainte hydrostatique $\sigma_{\text {hydr }}$ dépasse le module de Young en petites déformations de l'élastomère [15] soit :

$$
\sigma_{\text {hydr }}>5 / 6 E
$$

Si le matériau est considéré dans son domaine élastique linéaire (hypothèse raisonnable avant que cavitation ou fracture n'aient lieu), ces deux critères (la propagation de fracture et la cavitation) correspondent chacun à une valeur critique de déformation $\varepsilon$ définie comme :

$$
\varepsilon=\frac{h-h_{0}}{h_{0}}
$$

avec $h$ et $h_{0}$ les épaisseurs de la couche d'adhésif pendant et avant le décollement. Pour un $\alpha$ faible, le critère d'énergie sera atteint bien avant le critère de contrainte et on observera une propagation de fissure circulaire, alors que pour un $\alpha$ élevé, c'est le contraire qui se passe et la cavitation dans la couche confinée est favorisée. Cette transition peut être traduite par une carte d'amorçage des mécanismes de décollement en fonction de deux paramètres adimensionnels : le rapport $\alpha$ entre $a$ et $h$ et un paramètre adimensionnel d'adhésion $G_{\mathrm{c}} / E a$ entre le taux de restitution d'énergie critique $G_{\mathrm{c}}$ et le module d'élasticité $E$ de la couche de polymère.

Cette carte (Fig. 7) suggère que, lorsque $G_{\mathrm{c}}$ est grand et $E$ est faible (forte adhésion et faible module), la cavitation sera favorisée. Au contraire, lorsque le module $E$ et élevé et l'adhésion faible, la fracture interfaciale sera favorisée. Il est donc possible de modifier le mécanisme de rupture également en jouant sur ces deux paramètres $\left(G_{\mathrm{C}}\right.$ et $\left.E\right)$ en plus de la géométrie.

Toutefois, la figure 7 montre clairement que lorsque le degré de confinement est élevé et que le paramètre

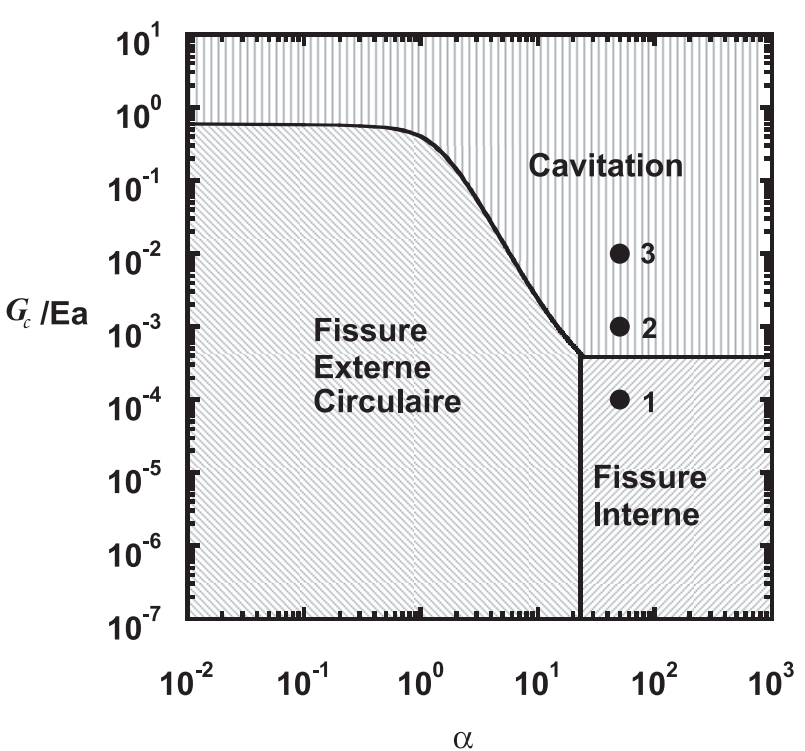

Fig. 7. Carte d'amorçage de fracture en fonction des deux paramètres adimensionnels $G_{\mathrm{c}} / E a$ et $\alpha$. Chaque zone correspond à un mécanisme d'amorçage de fracture différent. La cavitation est favorisée pour un $G_{\mathrm{c}}$ élevé et un module élastique faible, alors que la fracture est favorisée par une faible adhésion et un module élastique élevé. Dans tous les cas, une augmentation du degré de confinement favorise la cavitation à $G_{c} / E a$ fixé. Figure tirée de la référence [26].

$G_{\mathrm{c}} / E a$ n'est pas trop faible, c'est la cavitation qui est dominante $[19,26]$.

Ce type de carte ne constitue qu'un guide qualitatif au vu des hypothèses qui sont faites pour l'obtenir. Néanmoins, il prédit correctement la difficulté à propager une fissure lorsque la taille du système devient petite (contact d'une pointe d'AFM sur un matériau élastomère), le passage de la cavitation à la fracture lors d'une diminution de $G_{\mathrm{c}}$ à module constant [24] et le passage de la fracture à la cavitation lorsque le module élastique diminue à même valeur de $G_{\mathrm{c}}$ [28] . 
Pour compléter cette description, il faut encore mentionner que nous n'avons pas considéré ici la possibilité d'une fracture en volume dans la couche d'adhésif. En effet, pour des adhésifs de type autocollants utilisés dans nos expériences, elle n'est jamais observée. Toutefois, on peut imaginer une très bonne adhésion par des liaisons chimiques covalentes par exemple, et une faible résistance à la fracture, qui induirait une fracturation du matériau sans que le critère de cavitation ne soit atteint.

\section{Conclusions}

Nous avons discuté dans ce bref article l'effet d'un confinement sur les mécanismes d'endommagement des joints collés et plus généralement des couches polymère. Ces effets d'un confinement d'une couche polymère peuvent se résumer de la façon suivante :

Pour les matériaux hyperélastiques :

- la cavitation est favorisée comme mécanisme d'amorçage de la rupture;

- on passe d'un critère d'énergie critique de propagation de fissure à un critère de contrainte critique de cavitation en augmentant le confinement.

Pour les polymères vitreux ou semi-cristallins :

- les contraintes internes sont souvent modifiées par le confinement et la mixité de mode locale peut en être modifiée;

- les mécanismes de plasticité peuvent être modifiés là aussi pour favoriser la cavitation par rapport à la plasticité par bandes de cisaillement.

\section{Références}

[1] E.J. Kramer, Microscopic and Molecular fundamentals of crazing, Advances in Polymer Science 52/53 (1983) 1-56

[2] E.J. Kramer, L.L. Berger, Fundamental processes of craze growth and fracture, Advances in Polymer Science 91/92 (1990) 1-68

[3] J. Washiyama, C. Creton, E.J. Kramer, TEM fracture studies of polymer interfaces, Macromolecules 25 (1992) 4751-4758

[4] D.S. Dugdale, Yielding of steel sheets containing slits, J. Mechanics and Physics of Solids 8 (1960) 100-104

[5] H.R. Brown, A Molecular Interpretation of the toughness of glassy polymers, Macromolecules 24 (1991) 2752-2756

[6] C.Y. Hui, A. Ruina, C. Creton, E.J. Kramer, Micromechanics of crack growth into a craze in a polymer glass, Macromolecules 25 (1992) 3948-3955

[7] Y. Sha, C.Y. Hui, A. Ruina, E.J. Kramer, Continuum and discrete modeling of craze failure at a crack tip in a glassy polymer, Macromolecules 28 (1995) 2450-2459

[8] R. Schnell, M. Stamm, C. Creton, Direct correlation between interfacial width and adhesion in glassy polymers, Macromolecules 31 (1998) 2284-2292

[9] F. Kalb, L. Léger, C. Creton, C.J.G. Plummer, P. Marcus, A. Magalhaes, Molecular control of crack tip plasticity mechanisms at a PP-EPDM/PA6 interface Macromolecules 34 (2001) 2702-2709
[10] F. Kalb, Adhésion, Microstructure et microdéformations à l'interface de polymères semi-cristallins, Thèse Université Paris VI, Paris, 1998, 362

[11] N. Passade, Mécanismes de fracture d'interfaces polymères en géométrie confinée, Thèse Université Paris VI, Paris, 2000, 183

[12] C.J.G. Plummer, H.H. Kausch, C. Creton, F. Kalb, L. Léger, Structure and microdeformation of $(\mathrm{iPP} / \mathrm{iPP}-\mathrm{g}-$ MA)-PA6 reaction bonded interfaces, Macromolecules 31 (1998) 6164-6176

[13] J. Jiao, C.K. Gurumurthy, E.J. Kramer, Y. Sha, C.Y. Hui, P. Borgesen, Measurement of interfacial fracture toughness under combined mechanical and thermal stresses, J. Electronic Packaging 120 (1998) 349-353

[14] N. Passade, C. Creton, Y. Gallot, Fracture toughness of interfaces between glassy polymers in a trilayer geometry, Polymer 41 (2000) 9249-9263

[15] A.N. Gent, P.B. Lindley, Internal rupture of bonded rubber cylinders in tension, Proceedings of the Royal Society of London, series A: Mathematical and Physical Sciences, 249 A, 1958, pp. 195-205

[16] M.L. Williams, R.A. Schapery, Spherical flaw instability in hydrostatic tension, Inter. J. Fracture Mech. 1 (1965) 64-71

[17] J.F. Ganghoffer, A.N. Gent, Adhesion of a rigid punch to a thin elastic layer, J. Adhesion 48 (1995) 75-84

[18] Y.Y. Lin, C.Y. Hui, H.D. Conway, A detailed analysis of the flat punch (tack) test for pressure sensitive adhesives, J. Polymer Science: Part B: Polymer Physics 38 (2000) 2769-2784

[19] C. Creton, H. Lakrout, Micromechanics of flat probe adhesion tests of soft viscoelastic polymer films, J. Polymer Science: Part B: Polymer Physics 38 (2000) 965979

[20] K.R. Shull, C.M. Flanigan, A.J. Crosby, Fingering instabilities of confined elastic layers in tension, Phys. Rev. Lett. 84 (2000) 3057-3060

[21] D. Maugis, Contact, Adhesion and Rupture of Elastic Solids, Springer-Verlag, Berlin Heidelberg, New York, 1999

[22] G.H. Lindsey, Triaxial fracture studies, J. Appl. Phys. 38 (1967) 4843-4852

[23] A.N. Gent, Compression of rubber blocks, Rubber Chemistry and Technology 67 (1994) 549-558

[24] C. Creton, J.C. Hooker, K.R. Shull, Bulk and Interfacial Contributions to the Debonding Mechanisms of Soft Adhesives: Extension to Large Strains, Langmuir 17 (2001) 4948-4954

[25] K. Kendall, Adhesion: Molecules and Mechanics, Science 263 (1994) 1720-1725

[26] A.J. Crosby, K.R. Shull, H. Lakrout, C. Creton, Deformation modes of adhesively bonded elastic layers, J. Appl. Phys. 88 (2000) 2956-2966

[27] R.E. Webber, K.R. Shull, A. Roos, C. Creton, Effects of geometric confinement on the adhesive debonding of soft elastic solids, Phys. Rev. E 68 (2003) 021805

[28] G. Josse, De l'Adhérence à l'Anti-Adhérence à travers le Probe Tack, Thèse Université Paris VI, Paris, 2001, p. 250

[29] A. Chiche, Décollement d'adhésifs souples : cavitation et fracture, Thèse Université Paris VII, Paris, 2003

[30] H. Lakrout, C. Creton, D. Ahn, K.R. Shull, Influence of molecular features on the tackiness of acrylic polymer melts, Macromolecules 34 (2001) 7448-7458 